\title{
Article
}

\section{Activity of Oritavancin and Its Synergy with Other Antibiotics against Mycobacterium abscessus Infection In Vitro and In Vivo}

\author{
Gaoyan Wang ${ }^{1}$, Jia Tang ${ }^{1}$, Jiajia Feng ${ }^{1}$, Wenqi Dong ${ }^{1}$, Xinyu Huo ${ }^{1}$, Hao Lu ${ }^{1}$, Chenchen Wang ${ }^{1}$, Wenjia Lu ${ }^{1}$, \\ Xiangru Wang $1,2,3$, Huanchun Chen ${ }^{1,2,3}$ and Chen Tan $1,2,3, *$ \\ 1 State Key Laboratory of Agricultural Microbiology, College of Veterinary Medicine, Huazhong Agricultural \\ University, Wuhan 430070, China; 97wgy@webmail.hzau.edu.cn (G.W.); tangjia_6@163.com (J.T.); \\ 19fj@webmail.hzau.edu.cn (J.F.); $13137204926 @ 163 . c o m$ (W.D.); 2020302110145@webmail.hzau.edu.cn (X.H.); \\ sdluhao521@163.com (H.L.); 2018302110164@webmail.hzau.edu.cn (C.W.); hzaulwj1995@163.com (W.L.); \\ wangxr228@mail.hzau.edu.cn (X.W.); chenhch@mail.hzau.edu.cn (H.C.) \\ 2 Key Laboratory of Preventive Veterinary Medicine in Hubei Province, Wuhan 430070, China \\ 3 International Research Center for Animal Disease, Ministry of Science and Technology of the People's \\ Republic of China, Wuhan 430070, China \\ * Correspondence: tanchen@mail.hzau.edu.cn; Tel.: +86-027-8728-7170
}

Citation: Wang, G.; Tang, J.; Feng, J.; Dong, W.; Huo, X.; Lu, H.; Wang, C.; Lu, W.; Wang, X.; Chen, H.; et al. Activity of Oritavancin and Its Synergy with Other Antibiotics against Mycobacterium abscessus Infection In Vitro and In Vivo. Int. J. Mol. Sci. 2021, 22, 6346 .

https: / / doi.org/

10.3390/ijms22126346

Academic Editor: Sotiris

K. Hadjikakou

Received: 7 May 2021

Accepted: 9 June 2021

Published: 14 June 2021

Publisher's Note: MDPI stays neutral with regard to jurisdictional claims in published maps and institutional affiliations.

Copyright: (c) 2021 by the authors. Licensee MDPI, Basel, Switzerland. This article is an open access article distributed under the terms and conditions of the Creative Commons Attribution (CC BY) license (https:/ / creativecommons.org/licenses/by/ $4.0 /)$.

\begin{abstract}
Background: Pulmonary disease caused by Mycobacterium abscessus (M. abscessus) spreads around the world, and this disease is extremely difficult to treat due to intrinsic and acquired resistance of the pathogen to many approved antibiotics. M. abscessus is regarded as one of the most drug-resistant mycobacteria, with very limited therapeutic options. Methods: Whole-cell growth inhibition assays was performed to screen and identify novel inhibitors. The $\mathrm{IC}_{50}$ of the target compounds were tested against THP-1 cells was determined to calculate the selectivity index, and then time-kill kinetics assay was performed against M. abscessus. Subsequently, the synergy of oritavancin with other antibiotics was evaluated by using checkerboard method. Finally, in vivo efficacy was determined in an immunosuppressive murine model simulating $M$. abscessus infection. Results: We have identified oritavancin as a potential agent against M. abscessus. Oritavancin exhibited time-concentration dependent bactericidal activity against $M$. abscessus and it also displayed synergy with clarithromycin, tigecycline, cefoxitin, moxifloxacin, and meropenem in vitro. Additionally, oritavancin had bactericidal effect on intracellular M. abscessus. Oritavancin significantly reduced bacterial load in lung when it was used alone or in combination with cefoxitin and meropenem. Conclusions: Our in vitro and in vivo assay results indicated that oritavancin may be a viable treatment option against $M$. abscessus infection.
\end{abstract}

Keywords: Mycobacterium abscessus; oritavancin; synergy; immunosuppressive mice model

\section{Introduction}

M. abscessus, belonging to the rapidly growing mycobacteria, is an environmental pathogen that can cause chronic pulmonary infections in humans, especially in immunecompromised individuals, such as bronchiectasis and cystic fibrosis (CF) [1,2]. Additionally, the infection with $M$. abscessus results in accelerated damage to lungs, and it has been considered as a contraindication to lung transplantation [3,4]. Unfortunately, the inherent resistance of $M$. abscessus to some antibiotics, including several antituberculosis drugs, makes it one of the most resistant pathogens, posing a major threat to public health [5]. Except intrinsic resistance, adaptive and acquired resistances are also one of the important reasons why treatment of $M$. abscessus infection remains extremely difficult [6]. The US Cystic Fibrosis Foundation and the European Cystic Fibrosis Society have recommended several antibiotics such as macrolides, aminoglycosides, $\beta$-lactam, fluoroquinolones, and tetracyclines $[7,8]$. However, $M$. abscessus tends to develop acquired resistance to these antibiotics [9]. Besides antibiotics therapy, Rebekah et al. (2019) reported a phage therapy 
consisting of a cocktail of three engineered phages which was used to treat $M$. abscessus infection in a teenager afflicted with CF after bilateral lung transplantation [10].

Until now, there has been no official standard drug regimen for the treatment of $M$. abscessus infection. According to the latest guidelines of the American Thoracic Society and the Infectious Diseases Society of America (ATS/IDSA), it is suggested to use at least three active drugs among patients without macrolide resistance, and at least four active drugs among patients with macrolide resistance in the initial treatment phase (amikacin, imipenem (or cefoxitin), tigecycline, and azithromycin (clarithromycin), clofazimine, linezolid); and at least 2-3 active drugs for the continuation therapy phase (azithromycin (clarithromycin), clofazimine, linezolid, amikacin) [11,12]. However, current therapeutic regimens are usually accompanied by severe side effects [5]. Therefore, there is urgent medical need for more effective antibiotics treatment regimens to be developed for control this global threat.

Surprisingly, there are many compounds in clinical trials against $M$. tuberculosis, but few against $M$. abscessus [6]. As we know, development of novel antimicrobial drugs requires a complex and time-consuming verification process. Repurposed drugs may be a viable pathway for developing new treatment regimens. In the process of screening a library of drugs approved by the Food and Drug Administration for use against M. abscessus, we found that the lipoglycopeptide oritavancin possessed bacteriostatic activity against M. abscessus. Recently, several studies have reported that vancomycin and oritavancin show similar bacteriostatic activity against the clinically isolated M. abscessus complex, and there was synergy of vancomycin and clarithromycin against $M$. abscessus complex in vitro $[13,14]$. However, the therapeutic activity of oritavancin and vancomycin against $M$. abscessus in vivo remains largely unknown. In this study, we demonstrated that oritavancin exhibited activity against $M$. abscessus, both in vitro and intracellular. Oritavancin and clinically approved antibiotics displayed synergism in vitro. Moreover, oritavancin showed significant activity against $M$. abscessus in vivo when it was used alone or combined with cefoxitin and meropenem. Hence, our work may provide a viable therapeutic regimen against $M$. abscessus infection.

\section{Results}

\subsection{Activity of Oritavancin against M. abscessus and M. tuberculosis}

M. bovis BCG and $M$. smegmatis $\mathrm{mc}^{2} 155$ were commonly used as model strains to evaluate the antituberculosis activity of novel compounds. To determine the activity of oritavancin against $M$. abscessus and M. tuberculosis, the sensitivity of reference strains to oritavancin was tested by REMA (resazurin microtiter assay) method. As shown in Table 1 , oritavancin not only had an antibacterial effect on M. tuberculosis, but also exhibited activity against highly drug-resistant $M$. abscessus. The determination results of cytotoxicity of oritavancin against THP- 1 macrophages indicated that $\mathrm{IC}_{50}>128 \mu \mathrm{g} / \mathrm{mL}$ (Figure 1) and selectivity index (SI) $>16$ (for M. abscessus) and SI $>256$ (for M. tuberculosis H37Rv) $\left(\mathrm{SI}=\mathrm{IC}_{50} / \mathrm{MIC}\right)$ suggesting that oritavancin possessed a high selectivity to $M$. abscessus and M. tuberculosis.

Table 1. MICs and MBCs of oritavancin against M. abscessus and M. tuberculosis.

\begin{tabular}{ccc}
\hline & \multicolumn{2}{c}{ Concentration $(\mu \mathrm{g} / \mathrm{mL})$} \\
\hline Strains & MIC & MBC \\
\hline M. abscessus & 8 & 64 \\
M. tuberculosis H37Rv & 0.125 & 0.5 \\
M. tuberculosis H37Ra & 2 & 8 \\
M. bovis & 0.125 & 0.5 \\
M. bovis BCG & 0.5 & 1 \\
M. smegmatis $\mathrm{mc}^{2} 155$ & 8 & 32 \\
\hline
\end{tabular}




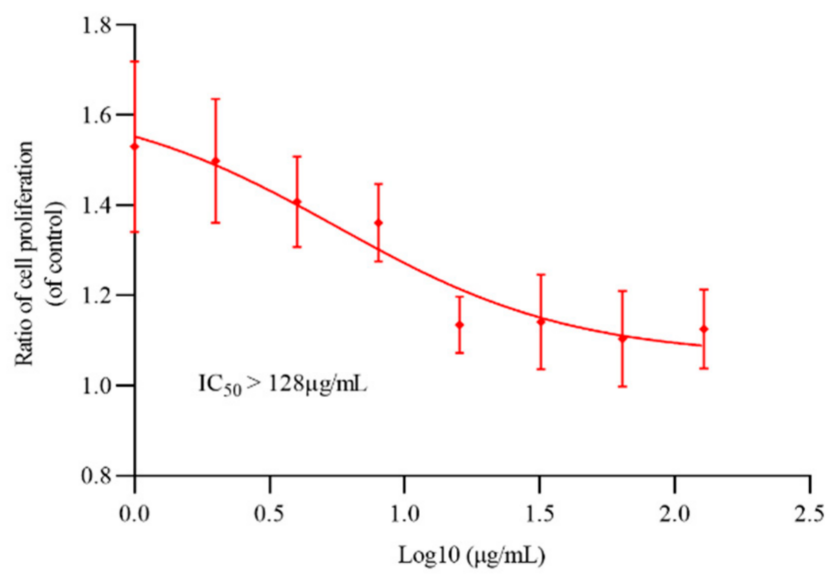

Figure 1. The cytotoxicity of oritavancin against THP-1 cells. The THP-1 cells were incubated with different concentrations of oritavancin for $72 \mathrm{~h}$ to determine its cytotoxicity. Data are expressed as the mean \pm standard deviation (SD) of triplicates for each concentration.

\subsection{Bactericidal Activity of Oritavancin against M. abscessus and M. bovis BCG In Vitro and Intracellular}

The potency of oritavancin $(1 / 2 \times$ MIC $8 \times$ MIC) killing M. abscessus and M. bovis BCG was evaluated. As shown in Figure 2a, $7.1 \log 10$ CFU and $5.6 \log 10$ CFU reduction was observed at day 5 post treatment with oritavancin at $8 \times$ MIC and amikacin at $4 \times$ MIC against $M$. abscessus, which was compared with untreated control. Moreover, no viable cells were recovered after 5-day treatments, suggesting limited probability of generating drug resistance. The 8-day treatment with oritavancin against $M$. bovis BCG at $4 \times$ MIC caused $8 \log 10 \mathrm{CFU}$ reduction (Figure $2 \mathrm{~b}$ ). In addition, the ability of oritavancin to clear intracellular infection of $M$. abscessus and $M$. bovis BCG was determined. The results indicated that at $8 \times \mathrm{MIC}$, oritavancin reduced the intracellular M. abscessus by $1.2 \log 10$ $\mathrm{CFU}$, whereas amikacin at $4 \times$ MIC reduced the cells by $0.9 \log 10 \mathrm{CFU}$ as compared with the control (Figure $2 \mathrm{c}$ ). The oritavancin (at $8 \times \mathrm{MIC}$ ) against $M$. bovis BCG resulted in $1.8 \log 10$ CFU reduction (Figure 2d). Taken together, our data indicate that oritavancin exhibited significant killing activity against M. abscessus and M. tuberculosis, in vitro and intracellular.

\subsection{Interactions with Other Clinically Utilized Antibiotics}

Single-drug treatments against $M$. abscessus are limited and rapidly led to drug resistance, therefore, drug combinations are necessary in the treatment of M. abscessus infection [15]. The combined effect of oritavancin and antibiotics utilized for clinical treatment was examined in vitro against $M$. abscessus by a checkerboard method. As shown in Table 2 and Figure 3, oritavancin exhibited a synergy with clarithromycin, tigecycline, and cefoxitin with $\sum$ FIC values of $0.5,0.141$, and 0.5 , respectively (Figure 3a-c). In addition, the combination of oritavancin with moxifloxacin and clarithromycin displayed synergy with $\sum$ FIC values of 0.5 and 0.226 (Figure 3d,e). More importantly, no antagonism between oritavancin and these antibiotics was observed, suggesting that oritavancin possessed the potential to be utilized for multidrug therapy treat $M$. abscessus infections. 
(a)

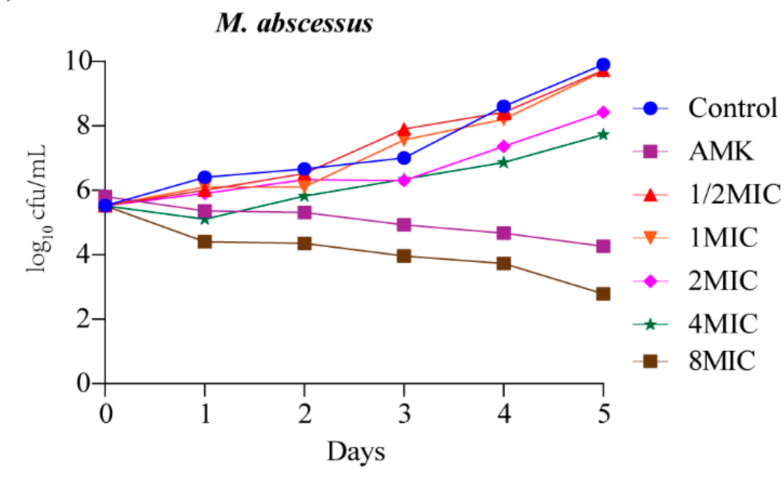

(b)

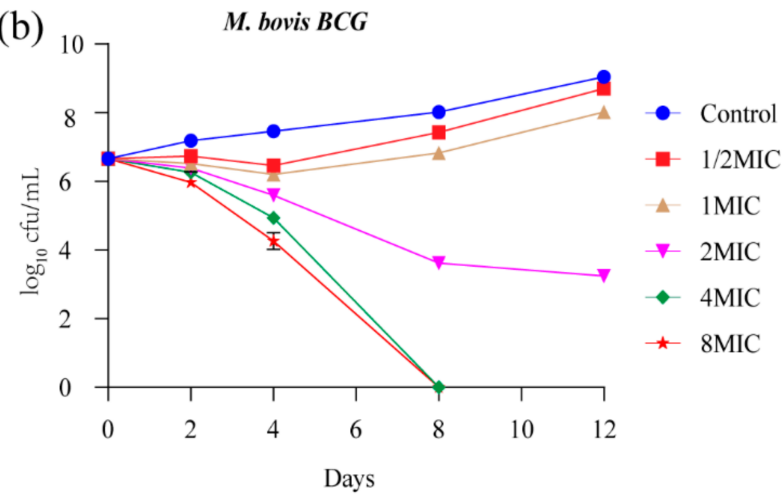

(c)

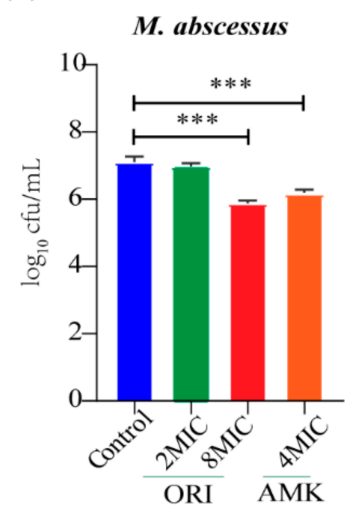

(d)

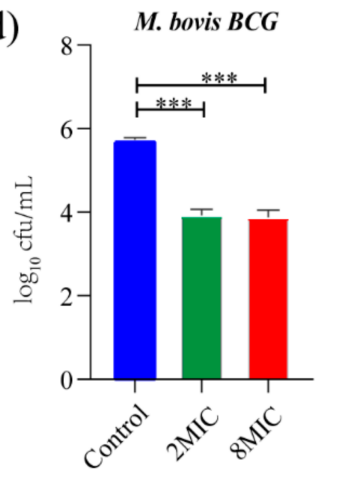

Figure 2. In vitro and intracellular bactericidal activity of oritavancin against M. abscessus and M. bovis BCG. Bactericidal activity of oritavancin against M. abscessus (a) and M. bovis BCG (b) was determined with different concentrations of oritavancin. And effect of oritavancin on M. abscessus (c) and M. bovis BCG (d) of viability in THP-1 cells was determined on $72 \mathrm{~h}$ after treatment with $2 \times$ MIC and $8 \times$ MIC oritavancin. Data are expressed as the mean \pm standard deviation (SD) of triplicates for each concentration. The error bar is smaller than the symbol size and not showed. ${ }^{* *} p<0.001$. ORI: oritavancin, AMK: amikacin.

Table 2. Synergy of oritavancin and approved antibiotics.

\begin{tabular}{cccccc}
\hline \multicolumn{7}{c}{ MIC $(\mu \mathrm{g} / \mathbf{m L})$} & & & \\
\hline Drug & Alone & Combination & FIC & $\sum$ FIC & Remarks \\
\hline Oritavancin & 8 & 2.0 & 0.25 & & \\
Clarithromycin & 0.0625 & 0.0156 & 0.25 & 0.5 & Synergism \\
Oritavancin & 8 & 0.125 & 0.0156 & & Synergism \\
Tigecycline & 1 & 0.125 & 0.125 & 0.141 & Synergism \\
Oritavancin & 8 & 4.0 & 0.25 & & \\
Cefoxitin & 4 & 1.0 & 0.25 & 0.5 & Synergism \\
Oritavancin & 8 & 4.0 & 0.25 & & Synergism \\
Moxifloxacin & 1 & 0.25 & 0.25 & 0.5 & No interaction \\
Oritavancin & 8 & 0.125 & 0.0156 & & \\
Meropenem & 4 & 1.0 & 0.25 & 0.266 & \\
Oritavancin & 8 & 1.0 & 0.125 & & No interaction \\
Levoxofloxacin & 2 & 1.0 & 0.5 & 0.563 & \\
Oritavancin & 8 & 0.5 & 0.0625 & 0.508 & \\
Amikacin & 8 & 4.0 & 0.5 & \multirow{2}{*}{0.625} & \\
Oritavancin & 8 & 2.0 & 0.125 & 0.625 & \\
\hline
\end{tabular}


Table 2. Cont.

\begin{tabular}{cccccc}
\hline \multicolumn{7}{c}{ MIC $(\mu \mathrm{g} / \mathbf{m L})$} & & & \\
\hline Drug & Alone & Combination & FIC & $\sum$ FIC & Remarks \\
\hline Bedaquiline & 0.25 & 0.125 & 0.5 & & No interaction \\
Oritavancin & 8 & 8 & 0.5 & 1.0 & \\
Linezolid & 0.25 & 0.125 & 0.5 & & No interaction \\
Oritavancin & 8 & 0.125 & 0.0078 & 1.01 & \\
Rifampicin & 1 & 1.0 & 1.0 & & No interaction \\
Oritavancin & 8 & 8.0 & 0.5 & 1.0 & \\
Imipenem & 16 & 8.0 & 0.5 & 0.5 & No interaction \\
\hline
\end{tabular}

(a)

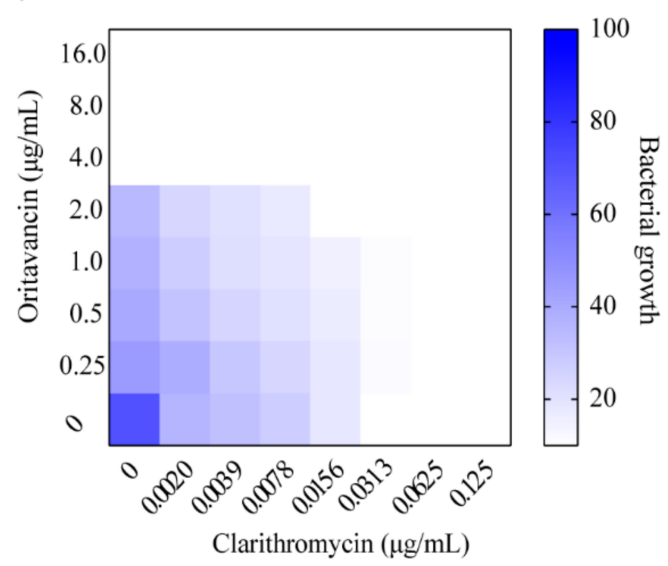

(c)

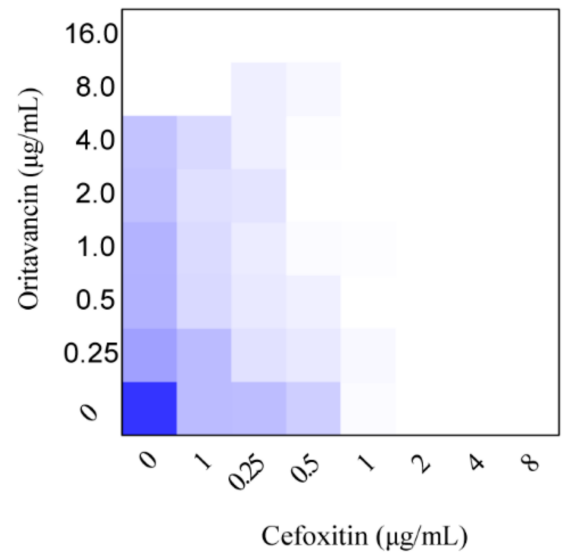

(d)

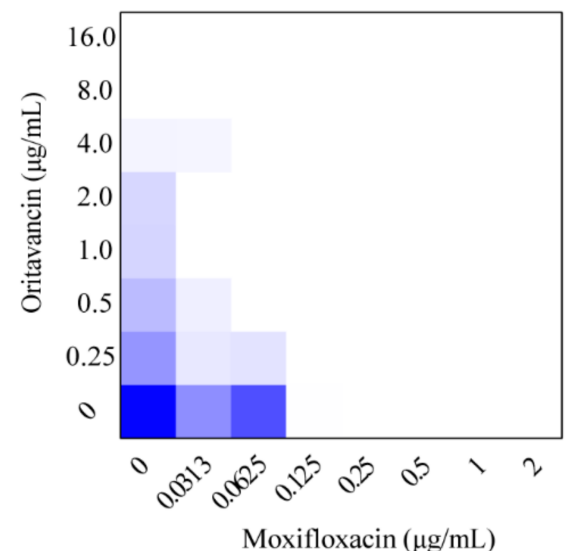

(b)

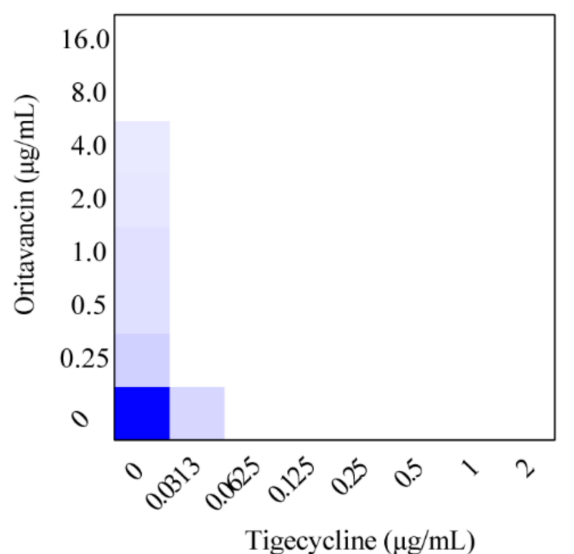

(e)

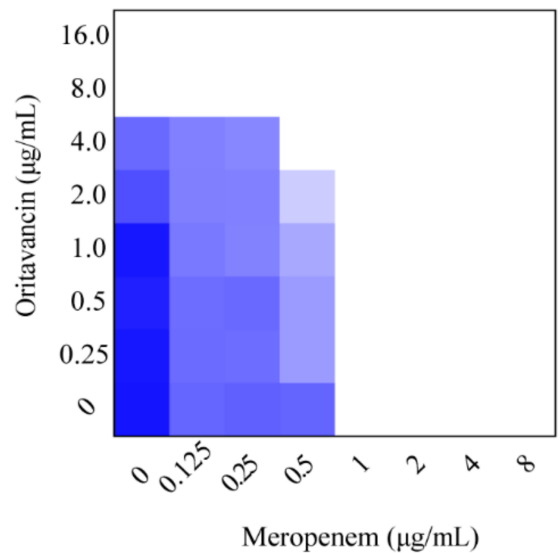

Figure 3. Synergism of oritavancin with (a) clarithromycin, (b) tigecycline, (c) cefoxitin, (d) moxifloxacin, and (e) meropenem against $M$. abscessus. Dark blue regions indicate high bacterial growth with low inhibition rate. The MICs of oritavancin, clarithromycin, tigecycline, cefoxitin, moxifloxacin, and meropenem against $M$. abscessus were $8.0 \mu \mathrm{g} / \mathrm{mL}, 0.0625 \mu \mathrm{g} / \mathrm{mL}$, $1.0 \mu \mathrm{g} / \mathrm{mL}, 4.0 \mu \mathrm{g} / \mathrm{mL}, 1.0 \mu \mathrm{g} / \mathrm{mL}$, and $4.0 \mu \mathrm{g} / \mathrm{mL}$, respectively. Data are expressed as the mean $\mathrm{OD}_{600}$ of two biological replicates. The FICI $\leq 0.05$ is defined as synergy.

2.4. M. abscessus-Killing Effect of Oritavancin in Combination with Clarithromycin, Cefoxitin, Moxifloxacin, Tigecycline, or Meropenem

Our in vitro MIC testing confirmed that oritavancin could synergize with other approved antibiotics. The result of time-kill kinetics assay of oritavancin against $M$. abscessus showed that oritavancin has no bactericidal effect at $1 \times$ MIC (Figure 2a). Therefore, we further explored whether the combination of oritavancin respectively with clarithromycin, cefoxitin, moxifloxacin, tigecycline, or meropenem at $1 \times$ MIC could improve the bactericidal 
activities of both antibiotics. As shown in Figure $4 a, b$, when oritavancin was in combination with clarithromycin or tigecycline, a $6 \log 10$ and $7.4 \log 10$ CFU decrease was observed on day 5 . In the case of the treatment with cefoxitin alone at $1 \times$ MIC, bactericidal effect was observed over the first 3 days of exposure, followed by an increasing CFUs afterwards. The treatment with moxifloxacin alone at $1 \times$ MIC exhibited a strong bactericidal effect on day 4 . The time-kill kinetics assay results of oritavancin at $1 \times$ MIC in combination of cefoxitin or moxifloxacin indicated that combined bactericidal effect of two antibiotics was superior to that of single one, Figure 4c,d. Furthermore, we found that treatment with meropenem alone at 1 $\times$ MIC had weak inhibitory activity, and that the combination of oritavancin with meropenem at $1 \times$ MIC resulted in complete killing on day 3 with no viable bacteria recovered after 2-day culture (Figure 4e). Thus, these results indicated the potential of oritavancin to be used as an adjuvant drug against $M$. abscessus infection.

(a)

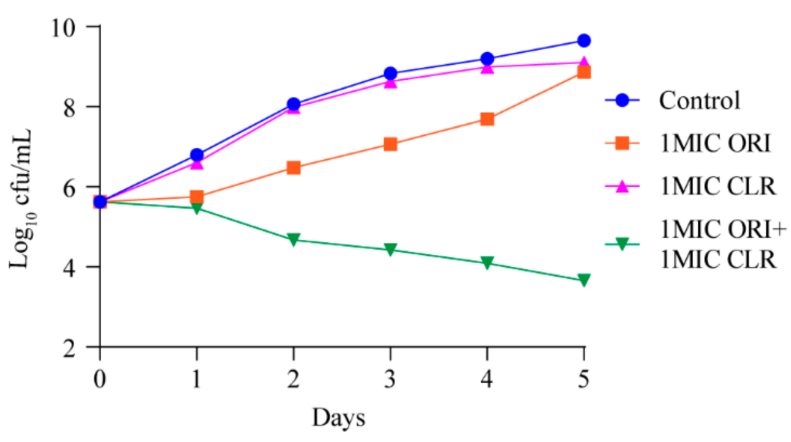

(c)

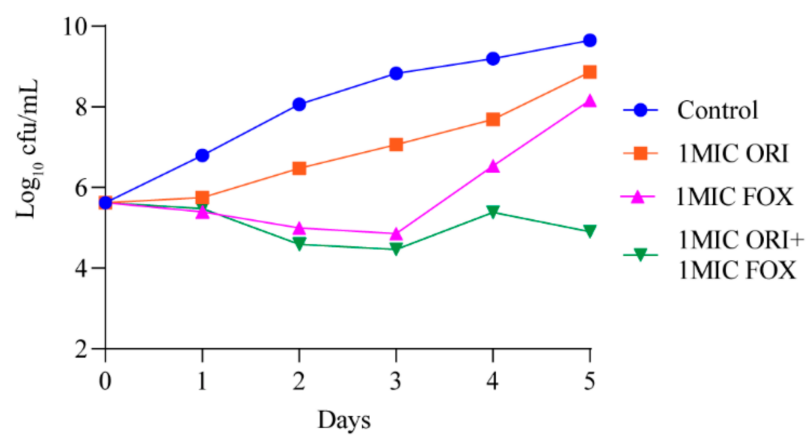

(e)

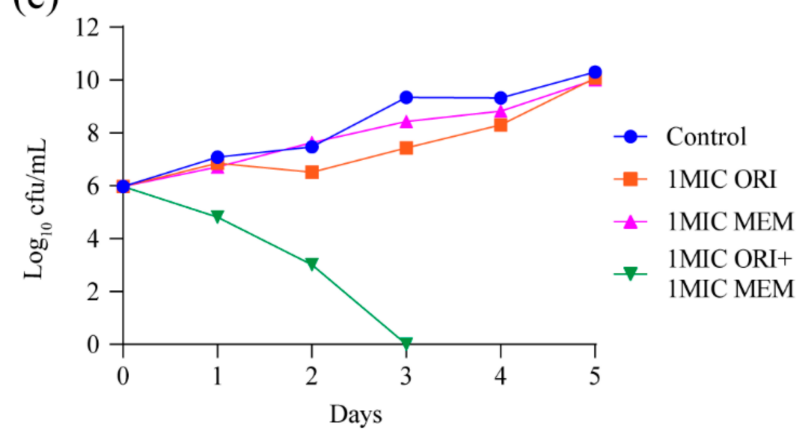

(b)

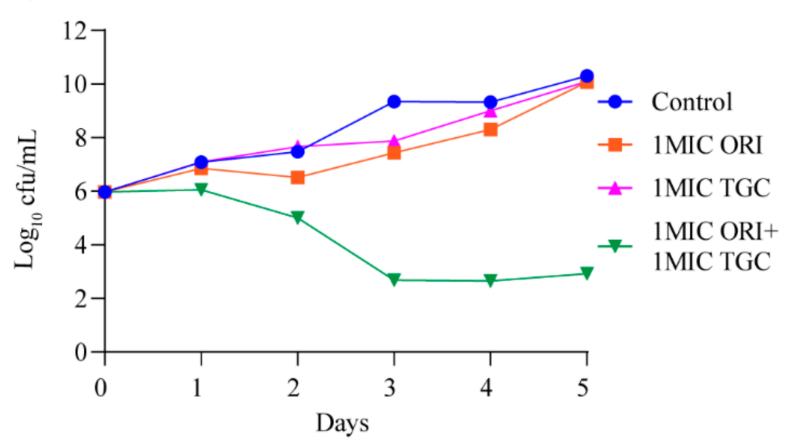

(d)

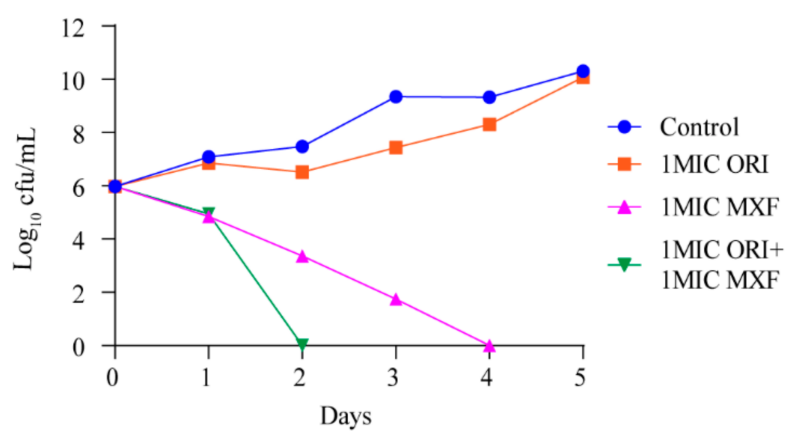

Figure 4. Oritavancin significantly potentiates (a) $1 \times$ MIC clarithromycin, (b) $1 \times$ MIC tigecycline, (c) $1 \times$ MIC cefoxitin, (d) $1 \times$ MIC moxifloxacin, and (e) $1 \times$ MIC meropenem bactericidal effect against M. abscessus in vitro. Data are represented the mean \pm standard deviation (SD) of triplicates for each concentration. The error bar is smaller than the symbol size and not showed. ORI, oritavancin; CLR, clarithromycin; TGC, tigecycline; FOX, cefoxitin; MXF, moxifloxacin; MEM, meropenem. 


\subsection{Activity of Oritavancin against M. abscessus Infection in Immunosuppressive Mouse Model}

Several $\beta$-lactams have been approved for the treatment of infection caused by $M$. abscessus in the United States and Europe $[8,16,17]$. Our results indicated in vitro synergy of oritavancin with cefoxitin, or meropenem against $M$. abscessus. The combined effect of these antibiotics against M. abscessus infection was further investigated in vivo. Thus, we utilized an immunosuppressive murine model to evaluate the therapeutic efficacy of these antibiotics in vivo (Figure 5a). As shown in Figure 5b, treatment with oritavancin (at $50 \mathrm{mg} / \mathrm{kg}$ ), cefoxitin (at $200 \mathrm{mg} / \mathrm{kg}$ ), and meropenem (at $100 \mathrm{mg} / \mathrm{kg}$ ) used caused an approximate reduction of $0.5 \log 10$ CFU in lungs, and clarithromycin (at $100 \mathrm{mg} / \mathrm{kg}$ ) alone led to a reduction of $0.9 \log 10 \mathrm{CFU}$, whereas oritavancin in combination with cefoxitin or meropenem resulted in reductions of $1.4 \log 10 \mathrm{CFU}$ and $1.3 \log 10 \mathrm{CFU}$ in lungs, respectively. In addition, a reduction of only $0.6 \log 10 \mathrm{CFU}$ in spleen was observed when oritavancin was combined with meropenem (Figure 5c). These results revealed that oritavancin had an effect similar to cefoxitin or meropenem in reducing bacterial load in the lungs of infected mice, and that the combination of oritavancin with cefoxitin or meropenem could significantly enhance the clearance of M. abscessus in the lungs.

(a)

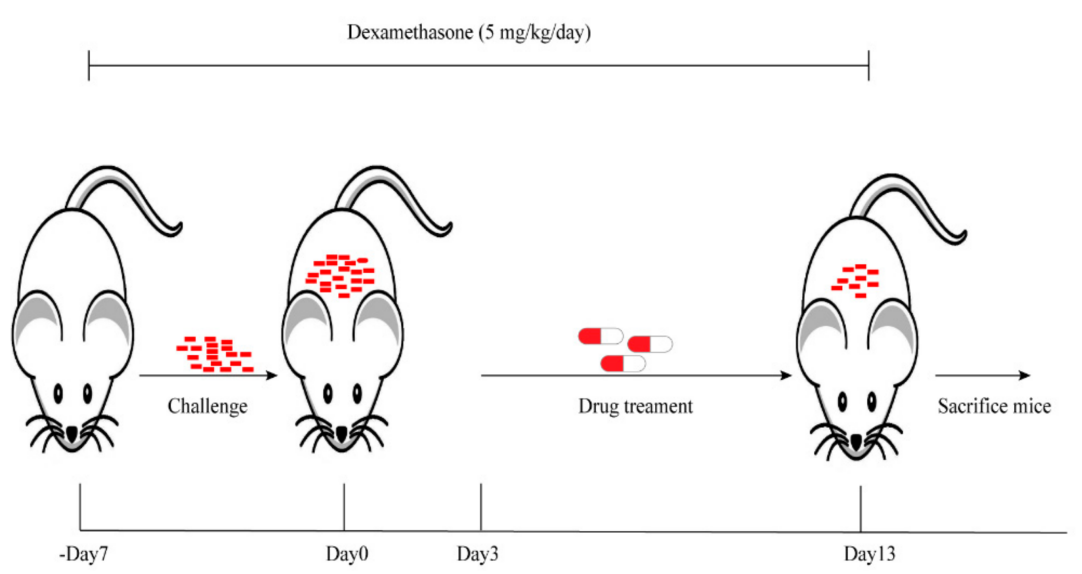

(b)

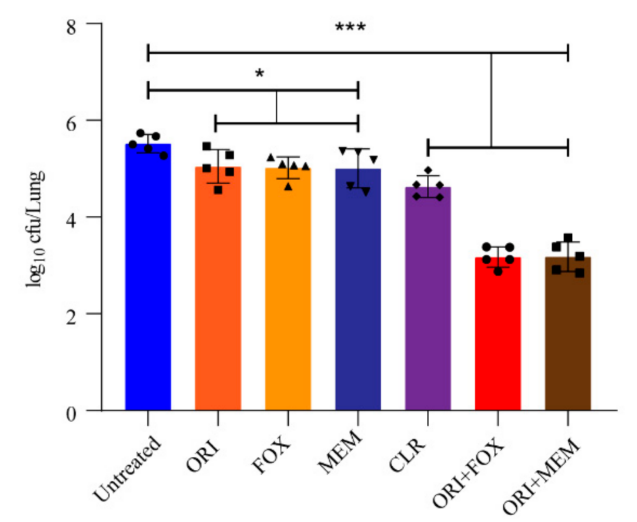

(c)

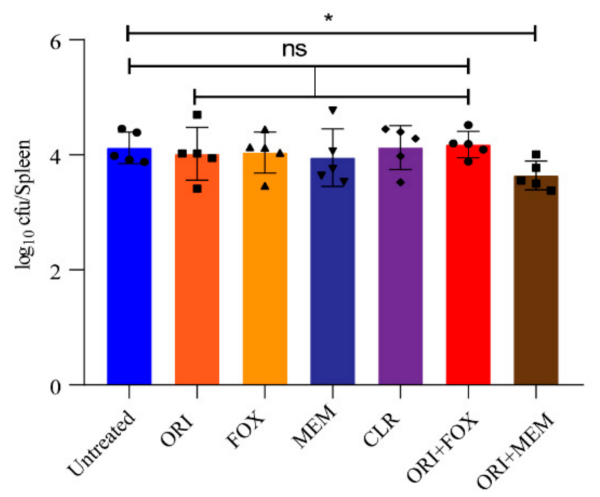

Figure 5. Evaluation of in vivo efficacy of oritavancin alone and its combination with cefoxitin or meropenem against $M$. abscessus in immunosuppressive mouse model. (a) Schematic diagram of mouse infection and drug treatment. -Day 7, 7 days before infection; Day 0, the day when challenge begins; Day 3, the day when infection ends and drug treatment starts; Day 13, the day when therapy ends and mice are sacrificed. The $\log 10 \mathrm{cfu} / \mathrm{mL}$ indicates bacterial load in lungs (b) and spleen (c) of infected mice after treatment with $50 \mathrm{mg} / \mathrm{kg}$ oritavancin (ORI), $200 \mathrm{mg} / \mathrm{kg}$ cefoxitin (FOX), and $100 \mathrm{mg} / \mathrm{kg}$ meropenem (MEM) alone or in combination. The treatment with $100 \mathrm{mg} / \mathrm{kg}$ clarithromycin (CLR) serves as positive control. ${ }^{*} p<0.05,{ }^{* * *} p<0.001 ;$ ns, not significant. 


\section{Discussion}

M. abscessus is a serious threat to human health which is naturally resistant to a broad range of antibiotics, and there is a lack of new active molecules. So far, treatment of M. abscessus infection has been particularly difficult, with a low cure rate $[6,18]$. Clarithromycin-based therapy regimens are less effective, and clarithromycin resistance in some isolates thus leads to treatment failure [19]. Therefore, finding novel antibacterial drugs to control M. abscessus infection is sorely needed. Currently, several different drug discovery approaches have been conducted, such as whole-cell screening, new combination studies that cause synergistic effects with already existing drugs or drug repositioning studies with old drugs [19]. Furthermore, conventional drug in new use offers a viable strategy for the clinical therapy of M. abscessus infection by screening anti-Mycobacterium abscessus drugs from an approved drug library. In this study, we found that oritavancin exhibited mild inhibition against $M$. abscessus. Oritavancin, as a new glycopeptide antibiotic, is active against several Gram-positive bacteria and pharmacokinetics characterized by extensive tissue distribution and a long terminal half-life, which may be an attractive option for the treatment of M. abscessus infection. It is effective in treating drug-resistant bacteria and clinical isolates [14]. Thus, the efficacy of oritavancin in the treatment of $M$. abscessus infections remains to be determined.

M. abscessus infections generally require treatment with multidrug combinations [20], oritavancin is active against several Gram-positive bacteria, which may be an attractive option for the treatment of M. abscessus infection. In the present study, oritavancin was active against M. abscessus, the MIC was $8 \mu \mathrm{g} / \mathrm{mL}$. We also found that oritavancin was effective in inhibiting the growth of intracellular $M$. abscessus in vitro at concentrations effective in inhibiting organisms growing extracellularly. However, the results of cytotoxicity showed that oritavancin has mild inhibition to THP- 1 cells at a concentration of $64 \mu \mathrm{g} / \mathrm{mL}$, which may interfere with the bactericidal effect of oritavancin on intracellular bacteria to a certain extent. We may be able to evaluate the pharmacological activity in other macrophages in future experiments, such as RAW264.7, J774A.1, etc. More importantly, the therapeutic activity of oritavancin was also evaluated in an immunosuppressive mouse model. Interestingly, oritavancin, with a quarter of the dose of cefoxitin or a half of the dose of meropenem, exhibited a similar activity to cefoxitin and meropenem in reducing bacterial load in the lungs of infected mice. Clarithromycin, the positive control, used alone showed better effectivity than the other three antibiotics (oritavancin, cefoxitin, and meropenem). However, when oritavancin was combined with cefoxitin or meropenem, it exhibited better bactericidal effect than clarithromycin. Even so, there is a flaw in our animal experiment. In future experiments, it is necessary to conduct further experiments in the mouse model to probe the synergistic effect of oritavancin with other known antibiotics at higher doses. As we know, glycopeptide antibiotics will damage the cell wall synthesis, thus they may facilitate drug penetration and enhance drug activity [21]. However, the ability of oritavancin is not absolute. The results of the combination showed that oritavancin only exhibited a synergism with clarithromycin, cefoxitin, meropenem, moxifloxacin, and tigecycline. To our surprise, when oritavancin was combined with cefoxitin and meropenem, such an effect was also observed in an immunosuppressive mouse model against $M$. abscessus infection. As far as we know, this is the first report of the combination between oritavancin and two $\beta$ - lactam antibiotics (cefoxitin and meropenem) against $M$. abscessus infection in vivo. Oritavancin exhibits single-use bactericidal activity and simultaneously enhances bacterial clearance, acting as an adjuvant. Nevertheless, extreme caution should be taken regarding the coadministration of oritavancin with other antibiotics in the clinical setting.

Data obtained in a phase III clinical trial, evaluating the effect of oritavancin as a targeted antibiotic for the treatment of patients with acute bacterial skin and skin structure infections, showed that an $138 \mu \mathrm{g} / \mathrm{mL}$ maximum plasma concentration was safely attained and well tolerated by patients $(n=297)$ administered with a single $1200 \mathrm{mg}$ dose [22]. The plasma concentration is higher than the MIC of oritavancin against $M$. abscessus in vitro determined in this study, which suggests that an effective dose can be achieved for the 
treatment of clinical M. abscessus infections. Even so, the clinical application of oritavancin required to be further determined.

Moreover, one important factor to consider is that oritavancin is administered intravenously [23]. This will be a limitation of using oritavancin considering the long clinical therapeutics duration of M. abscessus infections. Intravenous administration is a main concern associated with oritavancin, making it difficult to treat chronic diseases. Thus, modifying the drug-delivery mode may be necessary to consider. For example, Wilcox et al. reported that oritavancin, administered orally, exhibits significant activity against CDI in a hamster model in a dose-dependent manner [24]. Moreover, future study is suggested to provide the data of the antibacterial activity of oritavancin against clinical M. abscessus isolates. In conclusion, this study indicates that oritavancin exhibits activity against $M$. abscessus in vitro and in vivo, and it does not antagonize other most frequently used antibiotics for treating $M$. abscessus infections, suggesting the potential of oritavancin as a clinical drug for treating $M$. abscessus lung disease.

\section{Materials and Methods}

\subsection{Antimicrobial Agents}

Oritavancin diphosphate and bedaquiline were obtained from TargetMol (Shanghai, China). The others antibiotics were purchased from Sigma-Aldrich (Shanghai, China). All the compounds mentioned above were diluted in accordance with the recommendations of the manufacturers, divided into aliquots, and stored at $-20^{\circ} \mathrm{C}$.

\subsection{Bacterial Strains, Cell Line, and Culture Conditions}

M. abscessus ATCC19977, M. tuberculosis H37Rv ATCC27294, M. tuberculosis H37Ra ATCC25177, M. bovis ATCC19210, M. bovis BCG ATCC35737 (M. bovis BCG), and M. smegmatis $\mathrm{mc}^{2} 155$ ATCC700044 were propagated in Middlebrook 7H9 broth (BD, New York, NJ, USA) supplemented with 10\% OADC (BD, New York, NJ, USA), 0.2\% glycerol (Sigma, Saint Louis, MO, USA), and 0.05\% Tween 80 (Amresco, Houston, TX, USA) or on 7H11 agar plates supplemented with $0.5 \%$ glycerol and 10\% OADC. THP-1 macrophages (ATCC TIB-202) were cultured in RPMI-1640 medium (Gibco, Shanghai, China) supplemented with $10 \%$ fetal bovine serum (Gibco, Waltham, MA, USA) at $37^{\circ} \mathrm{C}$ with $5 \% \mathrm{CO}_{2}$.

\subsection{Mouse Experiments}

Animal experiments were performed on 6-week-old female BALB/c mice purchased from the Experimental Animal Center, Huazhong Agricultural University.

\subsection{Determination of Minimum Inhibitory Concentration (MIC) and Minimum Bactericidal Concentration (MBC)}

The MICs were determined using the previously described resazurin reduction microplate assay (REMA) method, with slight modifications [25]. Briefly, logarithmic bacteria were diluted to an $\mathrm{OD}_{600}$ of 0.01 . Two-fold serial dilutions of test compounds were performed in 96-well microplates. The MIC of each single antibiotic was tested in the panel with final drug concentrations ranging from 0.0625 to $128 \mu \mathrm{g} / \mathrm{mL}$. The microplates were added with $30 \mu \mathrm{L}$ of $0.01 \%$ resazurin solution per well and incubated for $24 \mathrm{~h}$ at $37^{\circ} \mathrm{C}$. A color change from blue to pink indicated bacterial growth. The minimum drug concentration that could prevent the color change was defined as MIC. MIC was determined at day 3 (for M. abscessus and M. smegmatis) and day 7 (for M. tuberculosis) post antibiotics incubation; $100 \mu \mathrm{L}$ of the inoculum from each well were 10-fold serially diluted and plated onto 7H11 agar plates to count the colony-forming units (CFUs). The minimum drug concentration that kills $99.9 \%$ of bacteria was defined as the MBC. The experiments were performed in triplicate. 


\subsection{Cytotoxicity Assay}

Cytotoxicity was evaluated with THP-1 cells using the WST-1 (Beyotime, Shanghai, China) assay. Cells were seeded into a 96-well microplate and incubated at $37^{\circ} \mathrm{C}$ with $5 \%$ $\mathrm{CO}_{2}$ for $12 \mathrm{~h}$. Different concentrations of oritavancin were added and incubated for $72 \mathrm{~h}$. The $10 \mu \mathrm{L}$ WST-1 solution was added into each well, and the microplate was incubated for another $2 \mathrm{~h}$. Thereafter, the $\mathrm{OD}_{450}$ was monitored using a fluorescence microplate reader for calculating the $50 \%$ inhibitory concentration $\left(\mathrm{IC}_{50}\right)$. The experiment was performed in triplicate.

\subsection{Intracellular Anti-Mycobacterial Activity}

THP-1 cells were seeded into 24-well plates and incubated with a final concentration of $100 \mathrm{nM}$ phorbol 12-myristate 13-acetate (PMA, AbMole, Shanghai, China) for $48 \mathrm{~h}$ to induce cell differentiation into macrophages. Cells were infected with $M$. abscessus at a MOI (multiplicity of infection) 4:1(cell: bacterium) for $1 \mathrm{~h}$ or $M$. bovis BCG at a MOI of 1: 20 (cell: bacterium) for $4 \mathrm{~h}$. The extracellular bacteria were removed by washing three times with PBS. Oritavancin was added to the corresponding well at $2 \times \mathrm{MIC}$ and $8 \times \mathrm{MIC}$ and $4 \times$ MIC of amikacin was also added to the well as a positive control. After $72 \mathrm{~h}$, the cell lysates were plated onto $7 \mathrm{H} 11$ agar plates to count CFUs. The experiments were performed in triplicate.

\subsection{Time-Concentration-Dependent Killing Assay}

Time-concentration-dependent killing abilities of oritavancin was determined as pre viously described [26]. Briefly, M. abscessus or M. bovis BCG with $\mathrm{OD}_{600}$ of 0.01 were cultured in 7H9 medium containing a range of $1 / 2 \times$ MIC to $8 \times$ MIC oritavancin. Amikacin, the positive control, was added to M. abscessus culture with $\mathrm{OD}_{600}$ of 0.01 to reach a final concentration of $4 \times$ MIC. M. abscessus was cultured for 5 days and M. bovis BCG for 12 days at $37^{\circ} \mathrm{C}$. At the indicated time, $100 \mu \mathrm{L}$ culture was serially diluted and plated onto $7 \mathrm{H} 11$ agar plates to count the CFUs.

For the combination of M. abscessus kill kinetic assays, oritavancin and its combinational antibiotics were added into $7 \mathrm{H} 9$ medium containing a final $\mathrm{OD}_{600} 0.01$ of $M$. abscessus to reach a final concentration of $1 \times$ MIC. As described above, the cultures were incubated for 5 days at $37^{\circ} \mathrm{C}$. During the 5-day incubation, cultures were serially diluted each day, and plated onto $7 \mathrm{H} 11$ agar plates. Subsequently, the resultant agar plates were incubated for 3 days at $37^{\circ} \mathrm{C}$, CFUs were counted. The experiments were performed in triplicate.

\subsection{Synergy Assay}

The synergic effect of oritavancin and other antibiotics against M. abscessus was determined using a checkerboard assay as described previously [27]. Briefly, a $50 \mu \mathrm{L}$ of serial dilution of oritavancin was added along the ordinate, and an equal volume of serial dilution of other antibiotics were added along the abscissa into 96-well microplate. The logarithmic phase of $M$. abscessus was diluted to an $\mathrm{OD}_{600}$ of 0.01 and then added to each well. Subsequently, microplates were incubated at $37^{\circ} \mathrm{C}$ for 3 days. After 3-day incubation, the $\mathrm{OD}_{600}$ of each well was measured with a Fluostar Omega microplate reader (SPARK $10 \mathrm{M}$, TECAN, Männedorf, Switzerland). The inhibition of $90 \%$ of bacterial growth was defined as synergy. The FICI was calculated using the formula:

$\mathrm{FICI}=\mathrm{FIC}_{\mathrm{A}}$ (MIC of drug A combination/MIC of drug A alone) $+\mathrm{FIC}_{\mathrm{B}}$ (MIC of drug $\mathrm{B}$ combination/MIC of drug B alone), $\mathrm{FICI} \leq 0.5$ indicated synergy; $0.5<\mathrm{FICI} \leq 4$ indicated no interaction; and FICI $>4$ indicated antagonism. The experiments were performed in duplicate.

\subsection{Bacterial Load Experiments}

Several studies have demonstrated that immunocompetent mice can gradually clear the infection of M. abscessus, whereas certain immunodeficient mice can maintain chronic infection in vivo [28,29]. Maggioncalda et al. reported that dexamethasone treatment can 
suppress the immune response of mice to sustain $M$. abscessus infection [28]. To obtain adequate immunosuppression and avoid interfering with the therapeutic effect of antibiotics in vivo by the mice's immune clearance, we treated the mice with dexamethasone to increase their susceptibility to an initial infection. Briefly, 6-week-old female BALB/c were randomly divided into seven groups (5 mice per group). Dexamethasone was administered by subcutaneous injection at $5 \mathrm{mg} / \mathrm{kg} /$ day (6 days per week), as previously described [30,31]. The administration of dexamethasone was initiated 1 week before infection and continued throughout the experiment. All mice were infected with $10^{6} \mathrm{CFU} \mathrm{M}$. abscessus via intravenous injection. From day 3 after infection, the infected mice were treated daily with antibiotics for 10 days. Clarithromycin was prepared in $10 \%$ hydroxyethyl- $\beta$ cyclodextrin and administered at a dose of $100 \mathrm{mg} / \mathrm{kg}$ by oral gavage to the mice as positive control. Oritavancin, cefoxitin, and meropenem were administered via subcutaneous injection at the doses of $50 \mathrm{mg} / \mathrm{kg}, 200 \mathrm{mg} / \mathrm{kg}$, and $100 \mathrm{mg} / \mathrm{kg}$, respectively. The doses of clarithromycin $(100 \mathrm{mg} / \mathrm{kg})$, cefoxitin $(200 \mathrm{mg} / \mathrm{kg})$, and meropenem $(100 \mathrm{mg} / \mathrm{kg})$ were described previously [29,30,32]. Then, the dose of oritavancin (50 mg/ $\mathrm{kg}$ ) was chosen, based on pharmacokinetic studies of oritavancin in mice; the peak plasma concentration of oritavancin was $228.83 \mu \mathrm{g} / \mathrm{mL}$ when oritavancin was intravenous at dose $20 \mathrm{mg} / \mathrm{kg}$ of body weight [33]. The serum concentration is higher than the MBC of oritavancin against $M$. abscessus in vitro determined here. Oritavancin, cefoxitin, meropenem, and clarithromycin were used for single-drug treatment. The drug combination treatment was as follows: oritavancin, cefoxitin, oritavancin and meropenem. The untreated group was treated with PBS. All the mice were sacrificed on day 10 after drug treatment, lungs and spleen were removed and homogenized. Homogenates were plated onto 7H11 agar plates to calculate CFUs for determining the bacterial loads of these organs.

\subsection{Ethical Approval}

The use of mice for infection experiment (HZAUMO-2021-0013) was approved by the Tab of Animal Experimental Ethical Inspection of Laboratory Animal Centre, Huazhong Agriculture University.

\subsection{Statistical Analysis}

For all experiments, a Student's $t$-test was used to evaluate differences between treated and control group and GraphPad Prism 8.0.1 software (GraphPad Software Inc., San Diego, CA, USA) was used to analyze data. Error bars indicate the standard deviation (SD) within the group. $p \leq 0.05$ was considered to be significant.

Author Contributions: C.T., H.C. and X.W. conceived and designed the experiments, G.W., J.T., J.F. and W.D. completed the antimicrobial and animal assays, X.H. performed the cytotoxicity assays, H.L., W.L. and C.W. analyzed and documented the data, G.W. wrote the paper. All authors have read and agreed to the published version of the manuscript.

Funding: This work was supported by grants from the National Key R\&D Program of China [2017YFD0500303] and the outstanding youth project of Natural Science Foundation in Hubei Province [2019CFA095].

Institutional Review Board Statement: The study was conducted according to the guidelines of the Declaration of Helsinki, and approved by the Tab of Animal Experimental Ethical Inspection of Laboratory Animal Centre, Huazhong Agriculture University (HZAUMO-2021-0013).

Informed Consent Statement: Not applicable.

Data Availability Statement: Data sharing not applicable. No new data were created or analyzed in this study. Data sharing is not applicable to this article.

Conflicts of Interest: The authors declare no conflict of interest. 


\section{References}

1. Winthrop, K.L.; McNelley, E.; Kendall, B.; Marshall-Olson, A.; Morris, C.; Cassidy, M.; Saulson, A.; Hedberg, K. Pulmonary nontuberculous mycobacterial disease prevalence and clinical features: An emerging public health disease. Am. J. Respir. Crit. Care Med. 2010, 182, 977-982. [CrossRef] [PubMed]

2. Moore, M.; Frerichs, J.B. An unusual acid-fast infection of the knee with subcutaneous, abscess-like lesions of the gluteal region; report of a case with a study of the organism, Mycobacterium abscessus, n. sp. J. Investig. Dermatol. 1953, 20, 133-169. [CrossRef] [PubMed]

3. Taylor, J.L.; Palmer, S.M. Mycobacterium abscessus chest wall and pulmonary infection in a cystic fibrosis lung transplant recipient. J. Heart Lung Transplant. 2006, 25, 985-988. [CrossRef] [PubMed]

4. $\quad$ Bryant, J.M.; Grogono, D.M.; Rodriguez-Rincon, D.; Everall, I.; Brown, K.P.; Moreno, P.; Verma, D.; Hill, E.; Drijkoningen, J.; Gilligan, P.; et al. Emergence and spread of a human-transmissible multidrug-resistant nontuberculous mycobacterium. Science 2016, 354, 751-757. [CrossRef]

5. Wu, M.L.; Aziz, D.B.; Dartois, V.; Dick, T. NTM drug discovery: Status, gaps and the way forward. Drug Discov. Today 2018, 23, 1502-1519. [CrossRef]

6. Johansen, M.D.; Herrmann, J.L.; Kremer, L. Non-tuberculous mycobacteria and the rise of Mycobacterium abscessus. Nat. Rev. Microbiol. 2020, 18, 392-407. [CrossRef]

7. Griffith, D.E.; Aksamit, T.; Brown-Elliott, B.A.; Catanzaro, A.; Daley, C.; Gordin, F.; Holland, S.M.; Horsburgh, R.; Huitt, G.; Iademarco, M.F.; et al. An official ATS/IDSA statement: Diagnosis, treatment, and prevention of nontuberculous mycobacterial diseases. Am. J. Respir. Crit. Care Med. 2007, 175, 367-416. [CrossRef]

8. Floto, R.A.; Olivier, K.N.; Saiman, L.; Daley, C.L.; Herrmann, J.L.; Nick, J.A.; Noone, P.G.; Bilton, D.; Corris, P.; Gibson, R.L.; et al. US Cystic Fibrosis Foundation and European Cystic Fibrosis Society consensus recommendations for the management of non-tuberculous mycobacteria in individuals with cystic fibrosis. Thorax 2016, 71 (Suppl. 1), i1-i22. [CrossRef]

9. Griffith, D.E. Mycobacterium abscessus and Antibiotic Resistance: Same As It Ever Was. Clin. Infect. Dis. 2019, 69, 1687-1689. [CrossRef]

10. Dedrick, R.M.; Guerrero-Bustamante, C.A.; Garlena, R.A.; Russell, D.A.; Ford, K.; Harris, K.; Gilmour, K.C.; Soothill, J.; Jacobs-Sera, D.; Schooley, R.T.; et al. Engineered bacteriophages for treatment of a patient with a disseminated drug-resistant Mycobacterium abscessus. Nat. Med. 2019, 25, 730-733. [CrossRef]

11. Daley, C.L.; Iaccarino, J.M.; Lange, C.; Cambau, E.; Wallace, R.J.; Andrejak, C.; Böttger, E.C.; Brozek, J.; Griffith, D.E.; Guglielmetti, L.; et al. Treatment of Nontuberculous Mycobacterial Pulmonary Disease: An Official ATS/ERS/ESCMID/IDSA Clinical Practice Guideline: Executive Summary. Clin. Infect. Dis. 2020, 71, e1-e36. [CrossRef]

12. Egorova, A.; Jackson, M.; Gavrilyuk, V.; Makarov, V. Pipeline of anti-Mycobacterium abscessus small molecules: Repurposable drugs and promising novel chemical entities. Med. Res. Rev. 2021. [CrossRef]

13. Mukherjee, D.; Wu, M.L.; Teo, J.W.P.; Dick, T. Vancomycin and Clarithromycin Show Synergy against Mycobacterium abscessus In Vitro. Antimicrob. Agents Chemother. 2017, 61. [CrossRef]

14. Chew, K.L.; Octavia, S.; Go, J.; Ng, S.; Tang, Y.E.; Soh, P.; Yong, J.; Jureen, R.; Lin, R.T.P.; Yeoh, S.F.; et al. In vitro susceptibility of Mycobacterium abscessus complex and feasibility of standardizing treatment regimens. J. Antimicrob. Chemother. 2020. [CrossRef]

15. Sarathy, J.P.; Ganapathy, U.S.; Zimmerman, M.D.; Dartois, V.; Gengenbacher, M.; Dick, T. TBAJ-876, a 3,5-Dialkoxypyridine Analogue of Bedaquiline, Is Active against Mycobacterium abscessus. Antimicrob. Agents Chemother. 2020, 64, e02404-19. [CrossRef]

16. Hamad, B. The antibiotics market. Nat. Rev. Drug. Discov. 2010, 9, 675-676. [CrossRef]

17. Lavollay, M.; Dubee, V.; Heym, B.; Herrmann, J.L.; Gaillard, J.L.; Gutmann, L.; Arthur, M.; Mainardi, J.L. In vitro activity of cefoxitin and imipenem against Mycobacterium abscessus complex. Clin. Microbiol. Infect. 2014, 20, O297-O300. [CrossRef]

18. Kwak, N.; Dalcolmo, M.P.; Daley, C.L.; Eather, G.; Gayoso, R.; Hasegawa, N.; Jhun, B.W.; Koh, W.J.; Namkoong, H.; Park, J.; et al. M ycobacterium abscessus pulmonary disease: Individual patient data meta-analysis. Eur. Respir. J. 2019, 54. [CrossRef]

19. Hanh, B.T.B.; Kim, T.H.; Park, J.W.; Lee, D.G.; Kim, J.S.; Du, Y.E.; Yang, C.S.; Oh, D.C.; Jang, J. Etamycin as a Novel Mycobacterium abscessus Inhibitor. Int. J. Mol. Sci. 2020, 21. [CrossRef]

20. Baines, S.D.; O'Connor, R.; Saxton, K.; Freeman, J.; Wilcox, M.H. Comparison of oritavancin versus vancomycin as treatments for clindamycin-induced Clostridium difficile PCR ribotype 027 infection in a human gut model. J. Antimicrob. Chemother. 2008, 62, 1078-1085. [CrossRef]

21. Reynolds, P.E. Structure, biochemistry and mechanism of action of glycopeptide antibiotics. Eur. J. Clin. Microbiol. Infect. Dis. 1989, 8, 943-950. [CrossRef] [PubMed]

22. Rubino, C.M.; Bhavnani, S.M.; Moeck, G.; Bellibas, S.E.; Ambrose, P.G. Population pharmacokinetic analysis for a single 1,200milligram dose of oritavancin using data from two pivotal phase 3 clinical trials. Antimicrob. Agents Chemother. 2015, 59, 3365-3372. [CrossRef] [PubMed]

23. Corey, G.R.; Kabler, H.; Mehra, P.; Gupta, S.; Overcash, J.S.; Porwal, A.; Giordano, P.; Lucasti, C.; Perez, A.; Good, S.; et al Single-dose oritavancin in the treatment of acute bacterial skin infections. N. Engl. J. Med. 2014, 370, 2180-2190. [CrossRef] [PubMed]

24. Wilcox Mark, H.; Baines, S.; Lehoux, D.; Parr Thomas, R. Method of Inhibiting Clostridium Difficile BY Administration of Oritavancin. U.S. Patent No. 8,518,873, 27 August 2013. 
25. Palomino, J.C.; Martin, A.; Camacho, M.; Guerra, H.; Swings, J.; Portaels, F. Resazurin microtiter assay plate: Simple and inexpensive method for detection of drug resistance in Mycobacterium tuberculosis. Antimicrob. Agents Chemother. 2002, 46, 2720-2722. [CrossRef] [PubMed]

26. de Steenwinkel, J.E.; de Knegt, G.J.; ten Kate, M.T.; van Belkum, A.; Verbrugh, H.A.; Kremer, K.; van Soolingen, D.; BakkerWoudenberg, I.A. Time-kill kinetics of anti-tuberculosis drugs, and emergence of resistance, in relation to metabolic activity of Mycobacterium tuberculosis. J. Antimicrob. Chemother. 2010, 65, 2582-2589. [CrossRef]

27. Zhang, Z.; Li, T.; Qu, G.; Pang, Y.; Zhao, Y. In vitro synergistic activity of clofazimine and other antituberculous drugs against multidrug-resistant Mycobacterium tuberculosis isolates. Int. J. Antimicrob. Agents 2015, 45, 71-75. [CrossRef]

28. Maggioncalda, E.C.; Story-Roller, E.; Ammerman, N.C.; Nuermberger, E.L.; Lamichhane, G. Progressive Mycobacterium abscessus lung infection in $\mathrm{C} 3 \mathrm{HeB} / \mathrm{FeJ}$ mice associated with corticosteroid administration. bioRxiv 2018, 418491. [CrossRef]

29. Lerat, I.; Cambau, E.; Roth Dit Bettoni, R.; Gaillard, J.L.; Jarlier, V.; Truffot, C.; Veziris, N. In vivo evaluation of antibiotic activity against Mycobacterium abscessus. J. Infect. Dis. 2014, 209, 905-912. [CrossRef]

30. Story-Roller, E.; Maggioncalda, E.C.; Lamichhane, G. Synergistic Efficacy of beta-Lactam Combinations against Mycobacterium abscessus Pulmonary Infection in Mice. Antimicrob. Agents Chemother. 2019, 63. [CrossRef]

31. Martins, T.G.; Trigo, G.; Fraga, A.G.; Gama, J.B.; Longatto-Filho, A.; Saraiva, M.; Silva, M.T.; Castro, A.G.; Pedrosa, J. Corticosteroidinduced immunosuppression ultimately does not compromise the efficacy of antibiotherapy in murine Mycobacterium ulcerans infection. PLoS Negl. Trop. Dis. 2012, 6, e1925. [CrossRef]

32. Hagihara, M.; Kato, H.; Sugano, T.; Okade, H.; Sato, N.; Shibata, Y.; Sakanashi, D.; Asai, N.; Koizumi, Y.; Suematsu, H.; et al. Pharmacodynamic evaluation of meropenem, cefepime, or aztreonam combined with a novel $\beta$-lactamase inhibitor, nacubactam, against carbapenem-resistant and/or carbapenemase-producing Klebsiella pneumoniae and Escherichia coli using a murine thigh-infection model. Int. J. Antimicrob. Agents 2021, 57, 106330. [CrossRef]

33. Boylan, C.J.; Campanale, K.; Iversen, P.W.; Phillips, D.L.; Zeckel, M.L.; Parr, T.R., Jr. Pharmacodynamics of oritavancin (LY333328) in a neutropenic-mouse thigh model of Staphylococcus aureus infection. Antimicrob. Agents Chemother. 2003, 47, 1700-1706. [CrossRef] 